\title{
Value Analysis of Transmission Distance in Urban Vehicular Networks
}

\author{
Ting Cao \\ Department of Computer Science and Engineering \\ Shanghai Jiao Tong University \\ Shanghai, China \\ vege_rabbit@yahoo.com.cn
}

\author{
Min-You Wu \\ Department of Computer Science and Engineering \\ Shanghai Jiao Tong University \\ Shanghai, China \\ mwu@sjtu.edu.cn
}

\begin{abstract}
IEEE $802.11 \mathrm{p}$ is a wireless communication protocol targeting at special requirements of vehicular communications. However, current study on IEEE 802.11p has not verified if the defined transmission distance is appropriate. Based on a discrete road model, we propose the concept of validity and necessity of the transmission distance, and then derive formula to calculate the probability to meet the requirements of validity and necessity with an approximate Poisson vehicle distribution. The formula is simulated based on real-world parameters of urban vehicular networks. The simulation result shows that validity and necessity of the transmission distance are relevant to the vehicle density. The transmission distance defined by IEEE 802.11p is unnecessarily large in order to meet the requirements of validity and necessity.
\end{abstract}

Keywords-IEEE 802.11p, transmission distance, urban vehicular network

\section{INTRODUCTION}

IEEE 802.11p [1] is also called WAVE (Wireless Access in the Vehicular Environment). It extends the traditional wireless short-range network technology, and tries to adapt to special requirements of vehicular communications.

IEEE 802.11p sets the maximum transmission distance to 1000 meters [2, 3]. However, current study on IEEE 802.11p has not verified if the pre-defined transmission distance is actually appropriate for urban vehicular networks.

On the one hand, if the transmission distance is too small, vehicular communications will not be guaranteed, which degrades the performance of applications in urban vehicular networks.

On the other hand, larger transmission distance leads to higher energy consumption and higher potential risks of data conflicts, which introduces higher management overheads of the network.

Thus, it is meaningful to identify the appropriate transmission distance for urban vehicular networks.

There are mainly two ways to analyze the transmission distance:

1) Set fixed values to the transmission distance. For example, Katrin Bilstrup [4] sets the transmission distance to 500 meters and 1000 meters.

2) Vary the transmission distance within a range. For example, J. Gozalves [5] sets the transmission distance to 0600 meters, 0-800 meters and 0-1200 meters.

In this paper, we first propose a discrete road model and the concept of validity and necessity of the transmission distance. Based on the definitions in Section II, we derive the formula to calculate the probability to meet the requirements of validity and necessity in Section III and analyze the formula in Section IV. Section V is the conclusion.

\section{MODELS AND DEFINITIONS}

\section{A. Road Model}

In our road model, a straight single-line road $R$ is evenly divided into $n$ slots, so that each slot can contain exactly one vehicle, as shown in Fig.1.

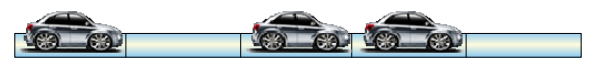

Figure 1. Road Model

We denote the road length as $L$ (kilometers), and the slot length as $l$ (kilometers).

It is obvious that the number of slots on the $\operatorname{road} R$ is $n=\frac{L}{l}$, and the number of slots on a road with unit length is $n_{0}=\frac{1}{l}$.

The entire urban road network can be seen as a mesh network consisting of a lot of roads described above.

\section{B. Transmission Distance}

According to the road model in the previous subsection, the distance between two vehicles is discretized into the number of slots for the first vehicle to reach the second one. For example, the distance between two vehicles in two adjacent slots is 1 .

The transmission distance is also discretized into the maximum number of slots that a data packet can be transferred towards a direction by a vehicle $v$. For example, if the transmission distance is 1 , the vehicle $v$ can only communicate with the vehicle in its adjacent slot.

\section{Validity and Necessity of Transmission Distance}

To determine if a transmission distance $k$ is appropriate, we define:

1) Validity: At any time $t$, each slot $i$ on the road $R$ must be at least within the transmission distance of one vehicle.

2) Necessity: For any transmission distance smaller than $k$, there are at least two vehicles on the road $R$ which are: 1 ) adjacent (there are no other vehicles inbetween); 2) not 
communicatable (the distance between the two vehicles is larger than the transmission distance).

If the transmission distance meets the validity requirement, a data packet can be transmitted from one vehicle to another on the road $R$ per one hop or multi-hops, so that the communication requirements of the applications in urban vehicular networks are satisfied.

If the transmission distance meets the necessity requirement, it reaches its minimum possible value, thus the energy consumption and the possibility of data conflicts are also minimized.

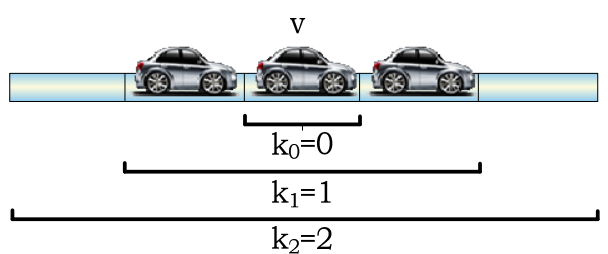

Figure 2. Validity and Necessity of Transmission Distance

For example, in Fig.2, the transmission distance $k_{0}$ does not meet the validity requirement; $k_{1}$ and $k_{2}$ both satisfy the validity requirement; however, only $k_{1}$ satisfies the necessity requirement at the same time. As a consequence, $k_{1}$ is the most appropriate transmission distance of the vehicle distribution in Fig.2.

\section{FORMULA CALCULATION}

In order to theoretically analyze validity and necessity of the transmission distance, we assume that the vehicle distribution on the $\operatorname{road} R$ follows a certain law, so that it is possible to derive a formula to calculate the probability to meet the requirements of validity and necessity.

Suppose all the vehicles advance one slot in one unit time period, and the probability that a vehicle enters the road $R$ in one unit time period is fixed and independent, then the probability that a slot on the road $R$ is occupied by a vehicle is also fixed and independent after $n$ time periods.

Since "the slot $i$ is occupied by a vehicle" is an independent event, the vehicle distribution on the road $R$ can be regarded as $n$ independently repeated trials, which means that the vehicle distribution on the road $R$ is an approximate Poisson distribution.

According to the road model in Section II, the probability that slot $i$ is occupied by a vehicle on the road $R$ is

$$
\frac{\rho}{n_{0}}=\frac{\rho}{1 / l}=\rho l
$$

The probability that slot $i$ is not occupied by a vehicle is

$$
p=1-\frac{\rho}{n_{0}}=1-\frac{\rho}{1 / l}=1-\rho l
$$

1) Original Fomula.

Let $A_{n}$ represents the event that "there are $k$ consecutive slots on the road $R$ that are unoccupied".

Let $F_{j}$ represents the event that "the first vehicle on the road $R$ appears in the $j^{\text {th }}$ slot”, and $H$ represents the event that "the first $k$ slots on the road $R$ are unoccupied", then $F_{1}$, $F_{2}, \ldots, F_{k}, H$ formulate a mutually exclusive complete event group.

According to Bayesian formula,

$$
P\left(\mathrm{~A}_{n}\right)=\sum_{j=1}^{k} P\left(\mathrm{~A}_{n} \mid \mathrm{F}_{j}\right) \mathrm{P}\left(\mathrm{F}_{j}\right)+\mathrm{P}\left(\mathrm{A}_{n} \mid \mathrm{H}\right) \mathrm{P}(\mathrm{H})
$$

a) For $P\left(\mathrm{~A}_{n} \mid \mathrm{F}_{j}\right)$, since the first vehicle appears in the $j^{\text {th }}$ slot and $j<k$, the first $j$ slots can no longer fulfill the requirement of $A_{n}$. Thus, the event is equivalently transformed to "there are $k$ consecutive slots in the last $n-j$ slots on the road $R$ that are unoccupied", so that we have

$$
P\left(\mathrm{~A}_{n} \mid \mathrm{F}_{j}\right)=\mathrm{P}\left(\mathrm{A}_{n-j}\right)
$$

b) For $\mathrm{P}\left(\mathrm{A}_{n} \mid \mathrm{H}\right), H$ already meets the requirement of $A_{n}$, so that we have

$$
\mathrm{P}\left(\mathrm{A}_{n} \mid \mathrm{H}\right)=1
$$

c) It is obvious that

$$
\begin{gathered}
\mathrm{P}\left(\mathrm{F}_{j}\right)=\mathrm{p}^{j-1}(1-\mathrm{p}) \\
\mathrm{P}(\mathrm{H})=\mathrm{p}^{k}
\end{gathered}
$$

Substitute (3) to (6) into (2), we have

$$
\begin{aligned}
P\left(\mathrm{~A}_{n}\right) & =\sum_{j=1}^{k} P\left(A_{n-j}\right) \mathrm{P}\left(\mathrm{F}_{j}\right)+\mathrm{P}(\mathrm{H}) \\
& =\sum_{j=1}^{k} P\left(A_{n-j}\right) \mathrm{p}^{j-1}(1-\mathrm{p})+\mathrm{p}^{k}
\end{aligned}
$$

It is obvious that $P\left(A_{n}\right)=0$ when $n<k$; and $P\left(A_{n}\right)=p^{k}$ when $n=k$.

Thus (9) can be rewritten as

$$
P\left(A_{n}\right)=\left\{\begin{array}{cc}
0, & n<k \\
p^{k}, \quad n=k \\
\sum_{j=1}^{n-k} P\left(A_{n-j}\right) p^{j-1}(1-\mathrm{p})+p^{k}, & n>k
\end{array}\right\}
$$

Then we can use recursion to calculate $P\left(A_{n}\right)$.

\section{2) Validity.}

Suppose $B_{n}$ represents the event that "there is at least one vehicle in any $k$ consecutive slots on the road $R$ ", which satisfies the validity requirement.

We have 


$$
\begin{aligned}
& P\left(\mathrm{~B}_{n}\right)=1-P\left(A_{n}\right) \\
& =\left\{\begin{array}{c}
1, \quad n<k \\
1-p^{k}, \quad n=k \\
1-\sum_{j=1}^{n-k} P\left(A_{n-j}\right) p^{j-1}(1-p)-p^{k}, \quad n>k
\end{array}\right\}
\end{aligned}
$$

3) Necessity.

Suppose $C_{n}$ represents the event that "there are $k-1$ consecutive slots on the road $R$ that are unoccupied". Then

$$
P\left(\mathrm{C}_{n}\right)=\left\{\begin{array}{cc}
0, & n<k-1 \\
p^{k-1}, \quad n=k-1 & \\
\sum_{j=1}^{n-k+1} P\left(C_{n-j}\right) p^{j-1}(1-\mathrm{p})+\mathrm{p}^{k-1}, & n>k-1
\end{array}\right\}
$$

Suppose $D_{n}$ represents the event that "there are $k$ consecutive slots on the road $R$, in which there is exactly one vehicle", which satisfies the necessity requirement. We have

$$
D_{n}=B_{n} C_{n}
$$

According to Bayesian formula,

$$
P\left(D_{n}\right)=P\left(B_{n} C_{n}\right)=P\left(B_{n}\right)-P\left(B_{n} C_{n}^{c}\right)
$$

Since $C_{n}^{c}$ represents the event that "there is at least one vehicle in any $k-1$ consecutive slots on the road $R$ ”, we have

$$
C_{n}^{c} \subset B_{n}(13)
$$

Thus,

$$
P\left(B_{n} C_{n}^{c}\right)=P\left(C_{n}^{c}\right)
$$

Substitute (14) into (12), we have

$$
\begin{aligned}
P\left(\mathrm{D}_{n}\right) & =\mathrm{P}\left(\mathrm{B}_{n}\right)-\mathrm{P}\left(\mathrm{C}_{n}^{c}\right) \\
& =P\left(B_{n}\right)+P\left(C_{n}\right)-1
\end{aligned}
$$

\section{VAlue ANAlysis of Transmission Distance}

In this section, we analyze the value range of the probability that meets validity and necessity of the transmission distance when the vehicle distribution follows the approximate Poisson distribution.

\section{A. Parameter Selection}

According to (1), (8) to (10) and (15), validity and necessity of the transmission distance is related to the vehicle density $\rho$, the slot length $l$, and the road length $L$.

\section{1) Vehicle Density.}

The vehicle amount and the road mileage in China's major cities can be found in China's major cities' statistical yearbooks.
It is obvious that vehicle density $=$ vehicle amount $/$ road mileage.

Assume at any time $t$ there are $50 \%$ of the total vehicles in the city driving on the road. Therefore, the actual vehicle density of the urban vehicular network should be coefficient weighted.

TABLE I. VEHICLE DENSITY OF ChINA’s MAJOR CitIES IN 2011

\begin{tabular}{|c|c|c|c|c|}
\hline City & $\begin{array}{c}\text { Vehicle } \\
\text { Amount } \\
\text { (thousand } \\
\text { vehicles) }\end{array}$ & $\begin{array}{c}\text { Road } \\
\text { Mileage } \\
\text { (kilomete } \\
\text { r) }\end{array}$ & $\begin{array}{c}\text { Vehicle } \\
\text { Density } \\
\text { (vehicles/ki } \\
\text { lometer) }\end{array}$ & $\begin{array}{c}\text { Weighted } \\
\text { Vehicle } \\
\text { Density } \\
\text { (vehicles/ki } \\
\text { lometer) }\end{array}$ \\
\hline Beijing & 3897 & 21319 & 182.79 & 91.40 \\
\hline Hangzhou & 1162.9 & 15418 & 75.42 & 37.71 \\
\hline Chongqing & 901.1 & 118600 & 7.60 & 3.80 \\
\hline Nanchang & 61 & 10573.26 & 57.69 & 28.85 \\
\hline
\end{tabular}

According to Tab.1, we choose the vehicle density as 5, 20,50 , and 100 vehicles per kilometer.

2) Slot Length.

TABLE II. VEHICLE LENGTH

\begin{tabular}{|c|c|c|}
\hline Manufacturer & Model & Length (meter) \\
\hline Shanghai Volkswagen Skoda & Octavia & 4.569 \\
\hline Import Kia & OPIRUS & 5.000 \\
\hline Import dodge & Kubo & 4.415 \\
\hline FAQ Mazda & Mazda 6 & 4.670 \\
\hline
\end{tabular}

According to the road model in Section II, one slot on the road $R$ can be occupied by exactly one vehicle. Based on the data in Tab.2, we choose 5 meters as the slot length $l$.

3) Road Length.

Tab.3 randomly picks one road in China's major cities, and electronically measures its length. According to the result, we choose the road length $L$ as 1 kilometer.

TABLE III. ROAD LENGTH OF CHINA’s MAJOR CitIES

\begin{tabular}{|c|c|c|}
\hline City & Road & Length (kilometer) \\
\hline Beijing & Qi'nian Avenue & 1.31 \\
\hline Hangzhou & Hubin Road & 0.87 \\
\hline Chongqing & Zhongshan Third Road & 0.52 \\
\hline Nanchang & Taoyuan Avenue & 1.00 \\
\hline
\end{tabular}

B. Value Analysis

We implement the calculation of (9) and (15) using the parameters picked in the section above.

1) Validity Analysis.

Fig. 4 shows that the validity probability curve $P\left(B_{n}\right)$ is roughly divided into two stages: at first fast rise, and then smooth transition; which means that the road connectivity changes rapidly in a short variation range of the transmission distance.

As can be seen from Fig.4, the larger the vehicle density is, the smaller the transmission distance satisfying the validity is. When the vehicle density is 5 vehicles per kilometer, the validity probability exceeds $90 \%$ at the transmission distance of 655 meters. However, when the 
vehicle density is 100 vehicles per kilometer, this number drops to 50 meters.

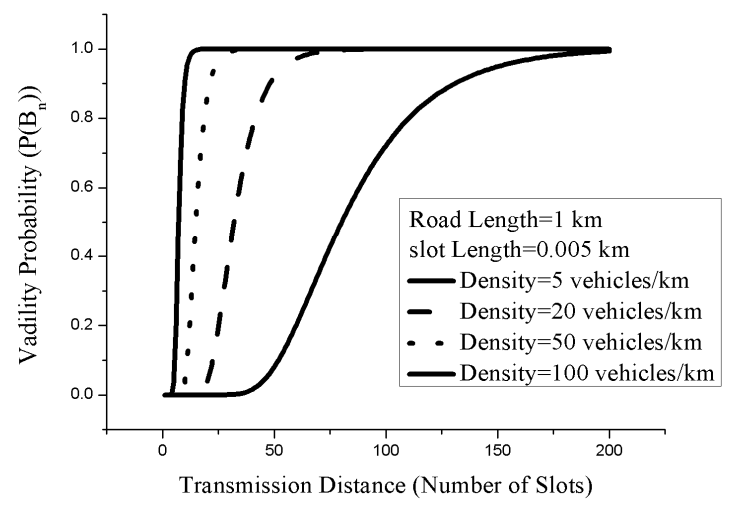

Figure 3. Validity Probability Curve $P\left(B_{n}\right)$

What's more, the larger the vehicle density is, the steeper the shape of the curve is. When the vehicle density is 5 vehicles per kilometer, the validity probability varies from $10 \%$ to $90 \%$ in 395 meters. However, when the vehicle density is 100 vehicles per kilometer, this happens within 25 meters.

Even in very sparse vehicular networks (vehicle density is 5 vehicles per kilometer), the transmission distance that fulfills the validity requirement is only 655 meters (where the validity probability reaches $90 \%$ ), which means that the transmission distance defined by IEEE 802.11p (1000 meters) is unnecessarily large in order to meet the validity requirement.

2) Necessity Analysis.

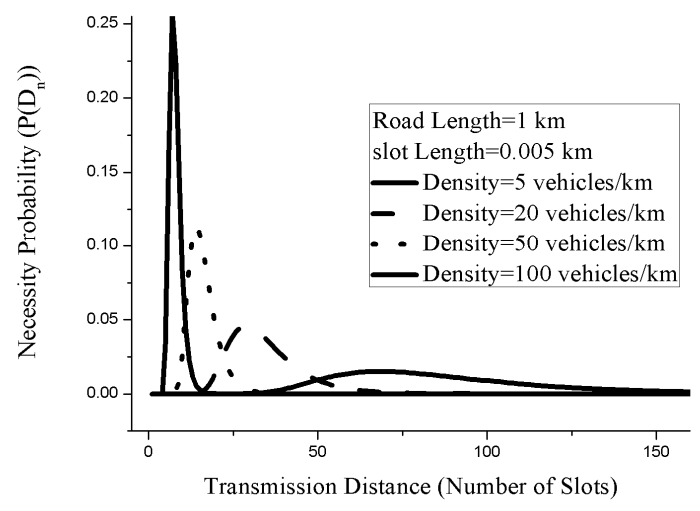

Figure 4. Necessity Probability Curve $P\left(D_{n}\right)$
Fig.5 shows that the necessity probability curve $P\left(D_{n}\right)$ is a pulse: the probability reaches the maximum value in a short variation range of the transmission distance, and then quickly drops back. The impact of the subsequent changes in the transmission distance becomes very small afterwards.

As can be seen from Fig.5, the larger the vehicle density is, the greater the maximum value of the necessity probability is, so that it is more likely that one transmission distance can satisfy the requirements of both validity and necessity at the same time.

For example, when the vehicle density is 5 vehicles per kilometer, the necessity probability reaches its maximum value at the transmission distance of 345 meters, and the corresponding validity probability is $33.8 \%$. However, when the vehicle density is 100 vehicles per kilometer, the maximum value appears at the transmission distance of 35 meters, and the corresponding validity probability is $45.6 \%$.

The maximum value of the necessity probability does not exceed $30 \%$, which means that it is hard to find a transmission distance that meets the requirement of both validity and necessity at the same time. The determined transmission distance should be a trade-off between validity and necessity.

\section{CONCLUSION}

In this paper, we analyze validity and necessity of the transmission distance in urban vehicular networks with approximate Poisson vehicle distribution. The validity and necessity probabilities vary with the vehicle density, and it is hard to meet both requirements at the same time. The transmission distance defined by IEEE 802.11p is unnecessarily large in order to meet the validity requirement.

\section{REFERENCES}

[1] L. M. S. C. o. t. I. C. Society, "IEEE standard for information technology-telecommunications and information exchange between systems-local and metropolitan area networks-specific requirements part 11: Wireless LAN medium access control (MAC) and physical layer (PHY) specifications amendment 6: Wireless access in vehicular environments," in IEEE Std 802.11p-2010 (Amendment to IEEE Std 802.11-2007 as amended by IEEE Std 802.11k-2008, IEEE Std 802.11r-2008, IEEE Std 802.11y-2008, IEEE Std 802.11n-2009, and IEEE Std 802.11w-2009, ed, July 2010.

[2] W. Fisher, "Development of DSRC/WAVE Standards," Annapolis: IEEE, 2007.

[3] V. S. C. Consortium, "Vehicle Safety Communications Report Task 3 Final Report: Identify Intelligent Vehicle Safety Applications enabled by DSRC," March 2005.

[4] K. Bilstrup, E. Uhlemann, E. G. Strom, and U. Bilstrup, "Evaluation of the IEEE 802.11 p MAC method for vehicle-to-vehicle communication," in Vehicular Technology Conference, 2008. VTC 2008-Fall. IEEE 68th, 2008, pp. 1-5.

[5] J. Gozalvez, M. Sepulcre, and R. Bauza, "IEEE 802.11 p vehicle to infrastructure communications in urban environments," Communications Magazine, IEEE, vol. 50, pp. 176-183, 2012. 